\title{
PROPOSIÇÕES DE CUIDADO CULTURAL À ENFERMAGEM FRENTE A ASPECTOS DA SAÚDE REPRODUTIVA DE MULHERES QUILOMBOLAS
}

\author{
CULTURAL CARE PROPOSALS FOR NURSING \\ REGARDING ASPECTS OF THE REPRODUCTIVE \\ HEALTH OF FEMALE MAROONS
}

\section{PROPUESTAS DE CUIDADOS CULTURALES PARA LA ENFERMERÍA DELANTE ASPECTOS DE LA SALUD REPRODUCTIVA DE LAS MUJERES CIMARRONES}

\author{
Amália Nascimento do Sacramento Santos ${ }^{1}$ \\ Enilda Rosendo do Nascimento ${ }^{2}$
}

\begin{abstract}
Como citar este artigo: Santos ANS, Nascimento ER. Proposições de cuidado cultural à enfermagem frente a aspectos da saúde reprodutiva de mulheres quilombolas. Rev baiana enferm. 2019;33:e33375.

Objetivo: descrever proposições de cuidado cultural para a enfermagem frente a aspectos da saúde reprodutiva de mulheres quilombolas rurais. Método: pesquisa com etnoenfermagem, realizada em comunidades quilombolas no estado da Bahia, Brasil, nos meses de janeiro a dezembro de 2014. Para coletar os dados, utilizou-se a Observação-Participação-Reflexão com registros em diário de campo e guia etnodemográfico. Participaram 25 mulheres quilombolas rurais que experimentaram gestação e parto. Os dados foram analisados com base na Teoria Transcultural do Cuidado. Resultados: muitos aspectos de cuidados alimentares, terapias complementares, conhecimento do corpo, relacionais, comunitários e políticos experimentados pelas mulheres quilombolas ampararam benefícios à saúde e outros potencializaram vulnerabilidades para o adoecimento. Conclusão: as proposições de cuidado cultural para a enfermagem frente a aspectos da saúde reprodutiva de mulheres quilombolas rurais podem favorecer maior empoderamento dessas mulheres e o enfrentamento de vulnerabilidades sociais.
\end{abstract}

Descritores: Enfermagem Transcultural. Saúde Reprodutiva. Saúde das Minorias Étnicas. Saúde da Mulher.

Objective: to describe proposals of cultural care for nursing regarding aspects of the reproductive bealth of rural female maroons. Method: research with ethnic nursing, held in maroon communities in the state of Babia, Brazil, in the months from January to December 2014. Data collection used the Observation-Participation-Reflection with records in the field diary and ethnic-demographic guide. The participants were 25 rural female maroons that have experienced pregnancy and childbirth. The data were analyzed based on the culture care theory. Results: many aspects of food care, complementary therapies, knowledge of the body, relations, community and policies experienced by female maroons sustained bealth benefits and others potentiated vulnerabilities for the illness. Conclusion: the cultural care proposals for nursing regarding aspects of the reproductive health of rural female maroons can promote greater empowerment of women and the coping of social vulnerabilities.

Descriptors: Transcultural Nursing. Reproductive Health. Health of Ethnic Minorities. Women's Health.

\footnotetext{
Enfermeira. Doutora em Enfermagem. Professora Adjunta da Universidade Federal do Recôncavo da Bahia. Santo Antonio de Jesus, Bahia, Brasil. amaliasacramento@hotmail.com. https://orcid.org/0000-0003-4536-867X

Enfermeira. Doutora em Enfermagem. Professora Titular Aposentada da Universidade Federal da Bahia. Salvador, Bahia, Brasil. https://orcid.org/0000-000 I-69933747
} 
Objetivo: describir las proposiciones de cuidado cultural para la enfermería delante aspectos de la salud reproductiva de las mujeres cimarrones rurales. Método: investigación con enfermería étnica, realizada en comunidades cimarrones en el estado de Babia, Brasil, en los meses de enero a diciembre de 2014. Para recopilar los datos, se utilizó la Observación-Participación-Reflexión con los registros en el diario de campo y guía étnico-demográfico. Participaron 25 mujeres cimarrones rurales que han experimentado el embarazo y el parto. Los datos fueron analizados con base en el cuidado transcultural, la teoría. Resultados: muchos aspectos del cuidado alimenticio, terapias complementarias, conocimientos del cuerpo, relacionales, comunitarios y políticos experimentados por mujeres cimarrones sostuvieron beneficios de salud y otros potenciaron las vulnerabilidades de la enfermedad. Conclusión: las proposiciones de cuidado cultural para la enfermería delante aspectos de la salud reproductiva de las mujeres cimarrones rurales pueden promover un mayor empoderamiento de la mujer y de afrontamiento de la vulnerabilidad social.

Descriptores: Enfermería Transcultural. Salud Reproductiva. Salud de las Minorías Étnicas. Salud de la Mujer.

\section{Introdução}

O cuidado às mulheres negras rurais quilombolas tem sido constatado como um desafio, com necessidades prementes de enfrentamento das vulnerabilidades sociais e ambientais a que estão expostas $^{(1)}$. Os cuidados profissionais em saúde reprodutiva prestados a essas mulheres, em muitas situações, não estão em sintonia com seus modos de vida e consideram seus saberes como marginais, ao reconhecerem apenas o cuidado biomédico ${ }^{(2)}$.

O avanço na melhoria da atenção pré-natal e seu reflexo na redução da mortalidade de mulheres no Brasil, notadamente durante a gravidez, têm possibilitado a identificação de desigualdades, quando se desagregam as informações por local de moradia, raça/cor da pele, renda, dentre outros aspectos. Observam-se diferenciais no pré-natal de mulheres brasileiras rurais e urbanas, em que as primeiras estão mais expostas às situações de vulnerabilidade em saúde. Esse quadro evidencia a persistência de desigualdades regionais e sociais para acesso a um cuidado adequado. Tem havido comprometimento da qualidade da assistência e dos cuidados na gestação, parto, puerpério e aborto, em especial para os contextos de ruralidade, como os da maioria das mulheres quilombolas ${ }^{(3)}$.

Além disso, as mulheres quilombolas experimentam o viés racial implícito. O racismo situa-se como um determinante social que potencializa suas dificuldades de acesso à saúde reprodutiva de qualidade. Este fato ocorre não só pela oportunidade de acesso, mas também pela possibilidade de distribuição de benefícios e oportunidades aos variados grupos, tendo como ponto de partida o caráter racial ${ }^{(4)}$. Evidenciaram-se desigualdades na evolução dos óbitos por hemorragia relacionados com gestação entre mulheres brancas e negras. No período de 2000 a 2012, esses óbitos decresceram em 34,05\% entre as mulheres brancas e aumentaram cerca de $6 \%$ na população de mulheres negras. Nesse contexto, afirma-se que as mulheres negras têm sido o alvo da mortalidade materna no Brasil ${ }^{(5)}$.

Em levantamento bibliográfico realizado em bases de dados da Scientific Electronic Library Online (SciELO) e da Literatura Latino-Americana e do Caribe em Ciências da Saúde (LILACS), utilizando os descritores "enfermagem transcultural", "etnoenfermagem" e "quilombolas", com um recorte para artigos brasileiros, entre os anos de 2004 e 2014, foram localizados 147 artigos, sendo 43 relacionados com a temática quilombolas e saúde. Poucos foram os estudos que tinham como ponto central a formulação de estratégias de cuidados em saúde reprodutiva de mulheres negras quilombolas. Por isso, torna-se relevante o desenvolvimento de estudos e pesquisas que tenham como ponto de convergência as estratégias de envolvimento de profissionais com a cultura de mulheres quilombolas, visto que a lógica global modernizante tem levado ao menosprezo de tradições culturais.

A competência cultural diz respeito à atitude de conseguir reconhecer e cuidar das pessoas em suas dinâmicas e problemas reais e/ou 
potenciais para acontecer. Para dar conta dessa competência, os profissionais devem valorizar os relacionamentos, desenvolver capacidade de escuta e manejar situações adversas, com trabalho multidisciplinar e projetos coletivos, com estabelecimento de parcerias e compromissos com as pessoas, respeitando seus modos de vida, sua família ${ }^{(6)}$. A definição de competência cultural assemelha-se com a de cuidado cultural abordado em estudo que discute diversidade cultural e universalidade do cuidado ${ }^{(7)}$.

Diante dessas considerações, o objetivo deste artigo é descrever proposições de cuidado cultural para a enfermagem frente a aspectos da saúde reprodutiva de mulheres quilombolas rurais.

\section{Método}

Trata-se de uma pesquisa exploratória, descritiva, com abordagem qualitativa, tendo como referencial teórico metodológico a Teoria Transcultural do Cuidado. Seguiram-se os critérios do Guia Internacional Consolidated Criteria for Reporting Qualitative Research (COREQ).

\section{A teoria transcultural do cuidado e o Modelo Sunrise}

Na teoria transcultural do cuidado, a autora $^{(7)}$ propôs um esquema operativo para sistematizar os conceitos teóricos que possibilitam conhecer o significado do cuidado nas diversas culturas e denominou-o Sunrise Model (Modelo Sunrise), simbolizado pelo nascer do sol (Figura 1).

Figura 1 - Modelo Sunrise proposto por Leininger

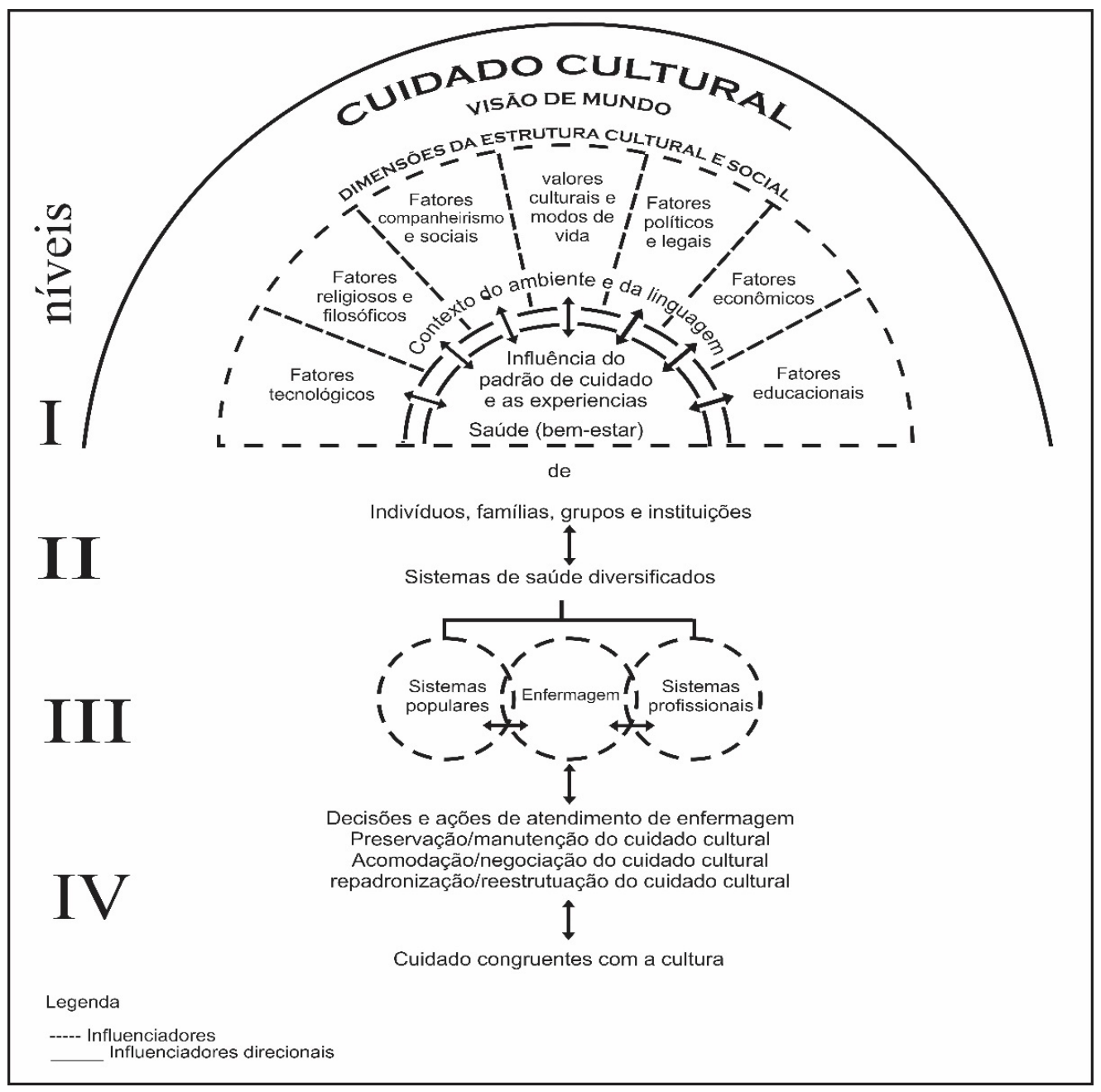

Fonte: Elaboração própria com base em Leopardi ${ }^{(8)}$. 
Observa-se que, esquematicamente, a figura apresenta duas partes: superior e inferior. A figura completa simboliza um círculo, a imagem do sol nascente, que representa o mundo da vida cotidiana, o universo de vida da pessoa e/ou comunidade. Na parte superior, estão representados fatores da visão de mundo e da estrutura da vida social que influenciam formas de cuidado. Esses fatores funcionam de forma interligada, de tal modo que sua influência no cuidado e na saúde acontecem por meio da linguagem e do contexto de ambiente. Na parte inferior da figura, observam-se os sistemas de saúde. Estes são discriminados como sistemas profissionais, sistemas populares e enfermagem. Nessa representação, a enfermagem deve conhecer e pesquisar todo esse universo - contexto de vida - para entender como acontece o cuidado humano e as implicações na saúde.

Observa-se, à esquerda da figura, os níveis de cuidado que vão do I ao IV. O primeiro nível possibilita verificar aspectos da vida social e o entendimento e as experiências de mundo das pessoas. O segundo, permite descobertas sobre as formas de cuidado no que tange aos aspectos individuais, das famílias, e a cultura do serviço de saúde, atendo-se às descobertas das suas expressões e dos seus significados. Nesse caso, necessita-se estar imerso na cultura para obter um conhecimento autêntico daquela realidade. No terceiro nível, o foco reside nas diferenças e semelhanças entre os sistemas de saúde populares e profissionais.

Finalmente, o quarto nível objetiva o desenvolvimento de cuidado de enfermagem e deve ser referendado, valorizado e relacionado com a cultura. A identificação de seus aspectos representa a construção de um cuidado congruente com a cultura. Nessa perspectiva, discutem-se três possibilidades de ação: preservação e/ou manutenção do cuidado cultural, que diz respeito às ações profissionais que colaboram com pessoas de determinada cultura para preservar valores relevantes do cuidar, de modo a enfrentar dificuldades e manter o bem-estar; acomodação e/ou negociação do cuidado cultural, que se refere às ações profissionais que colaboram com as pessoas de uma cultura com base em negociações, isto é, discussões compartilhadas, de detalhes do cuidado popular que possam ser reorganizados para um resultado de saúde mais benéfico; e, por fim, a Repadronização ou Reestruturação do cuidado cultural, que explicita ações profissionais que colaboram com as pessoas de uma cultura de modo a reordenar, mudar seus modos de vida para padrões de cuidados novos e benéficos, de maneira coparticipante, instituindo melhores padrões de cuidado, práticas ou resultados ${ }^{(7)}$.

\section{Procedimentos metodológicos}

O estudo foi realizado entre os meses de janeiro e dezembro de 2014, em uma comunidade quilombola do Recôncavo da Bahia, Brasil, caracterizada pela situação de ruralidade e solo de manguezal. As principais fontes econômicas e de subsistência são o extrativismo vegetal, destacando o cultivo de mandioca e do dendê, e o trabalho de pesca, principalmente de mariscos. Esta é a principal atividade econômica desenvolvida pelas mulheres, que se acumula com o trabalho do cuidado não remunerado, desenvolvido no âmbito familiar/doméstico.

A entrada no campo e o contato com as participantes do estudo foi precedida por contato direto com representantes do Conselho Quilombola local e pela participação da pesquisadora em suas reuniões.

Participaram do estudo informantes-chave e informantes gerais. Os critérios de inclusão de informantes-chave foram: ser mulher quilombola; residir na comunidade do estudo; e já ter vivenciado os eventos reprodutivos de gestação e parto. Para informantes gerais, os critérios foram: ser pessoa conhecida na comunidade; realizar cuidados com mulheres nos eventos reprodutivos (agentes de saúde, profissionais da unidade de saúde da família local, rezadeiras, parteiras, responsáveis pelas religiões de matrizes africanas). Os critérios de exclusão para ambos os participantes foram: não apresentar condições físicas e/ou mentais para a conversação; mulheres adolescentes não autorizadas 
pela pessoa responsável para participar do estudo. As informantes-chave e gerais foram selecionadas com base na técnica de bola de neve, iniciada com indicação de lideranças locais.

Foram utilizadas as técnicas da observação sistemática, tendo como instrumento o diário de campo e um guia etnodemográfico. A análise dos dados foi realizada em quatro etapas propostas no método da etnoenfermagem ${ }^{(7)}$ : apreensão dos dados (refere-se à coleta e documentação dos dados brutos, com identificação de palavras-chave relativas ao problema da pesquisa); síntese (identificação dos descritores e indicadores identificados no aprofundamento de leituras dos dados); teorização (análise contextual e de padrões atuais, interpretando-se as categorias de acordo com o referencial teórico); e recontextualização (identificação dos temas e dos achados relevantes da pesquisa, além da articulação dos resultados com a literatura científica).

Os dados extraídos da observação participante, da reflexão e do guia etnodemográfico permitiram a construção de três quadros que expõem resumos categorizados com base na proposição da Teoria Transcultural do Cuidado, no que tange aos modos de decisões e ações de enfermagem para o cuidado congruente com a cultura, designando-os para a ação de preservação, negociação ou reestruturação ${ }^{(7)}$. Por fim, essas três categorias temáticas foram discutidas com base em multirreferências.

Assim, os cuidados e os aspectos que eram valorizados pelas mulheres e expressavam benefícios para a saúde e para a vida em comunidade foram descritos em um quadro denominado "Ações para Preservação do Cuidado". Os cuidados ou aspectos valorizados pelas mulheres como potencialmente benéficos, mas apresentavam interface com vulnerabilidades para adoecimento, foram elencados no modo de ação de negociação; os aspectos que significavam dificuldades e vulnerabilidades, sofrimentos ou problemas e não expressavam benefícios para a vida e a saúde foram incluídos no modo de ação de reestruturação de cuidados.

Os princípios éticos em pesquisas envolvendo seres humanos foram observados em conformidade com a Resolução n. 466/2012, do Conselho Nacional de Saúde. Obteve-se anuência da Secretaria Municipal de Saúde local e do Conselho Quilombola. O projeto foi aprovado por Comitê de Ética em Pesquisa, CAAE número 29433014.1.0000.5531.

\section{Resultados e Discussão}

Apresenta-se brevemente a caracterização socioeconômico das mulheres estudadas e as proposições de cuidados, quais sejam manutenção, negociação e repadronização do cuidado cultural, com base em aspectos observados nos modos de vida das participantes.

\section{Caracterização socioeconômica das mulheres}

As mulheres participantes possuíam baixa escolaridade, a maioria não concluíra o ensino fundamental e algumas eram analfabetas; possuíam companheiro com união estável; e conviviam no domicílio com uma média de cinco pessoas. Todas se autodeclararam negras. As mulheres, em sua maioria, apresentavam baixa condição socioeconômica. As fontes de renda e de subsistência eram o trabalho agrícola, a pesca, com produção de mariscos, e os benefícios sociais, como o Bolsa Família, Bolsa Verde e Bolsa Escola. Politicamente, a comunidade estava organizada por meio de conselhos e associações de moradores, que se reuniam sistematicamente. Essas características assemelham-se às de outras mulheres quilombolas brasileiras, denotando um perfil de vulnerabilidade em saúde e, ao mesmo tempo, seus traços de resistência e de enfrentamentos resilientes ${ }^{(1)}$.

\section{Proposições de preservação e/ou manutenção do cuidado cultural}

Aponta-se, no Quadro 1, aspectos culturais observados na comunidade, consideradas as potencialidades, tanto na perspectiva êmica, visto que as mulheres valorizavam algumas habilidades e cuidados próprios e revelavam que eram 
muitas vezes as únicas formas de cuidado que se lhes apresentavam, quanto na perspectiva ética, pois entende-se que são cuidados efetivos, na medida em que resolvem ou podem ajudar a resolver ou atenuar problemas de saúde e/ou sociais. Em seguida, apresentam-se algumas considerações contextuais, com a finalidade de explicar a decisão de propor a preservação.

Quadro 1 - Aspectos culturais do cuidado popular em saúde reprodutiva de mulheres quilombolas com reflexões para ação de preservação e/ou manutenção do cuidado

\begin{tabular}{|c|c|}
\hline Subcategorias & Aspectos culturais observados \\
\hline $\begin{array}{l}\text { Cuidados } \\
\text { alimentares }\end{array}$ & $\begin{array}{l}\text { Hábitos alimentares para prevenir anemia, utilizando vegetais com maior teor de } \\
\text { ferro, como quiabo, beterraba, feijão, couve, jenipapo; dieta do pós-parto com } \\
\text { alimentos restritivos; restrição do uso de pimenta na gestação, evitando parto } \\
\text { prematuro e abortos. }\end{array}$ \\
\hline $\begin{array}{l}\text { Terapias } \\
\text { complementares }\end{array}$ & $\begin{array}{l}\text { Rezas e benzimentos na gestação; uso de ervas fitoterápicas na forma de } \\
\text { banhos e chás na gestação e parto. As mulheres colhem ou compram plantas } \\
\text { fitoterápicas, tais como flor de algodão, quiôiô, aroeira, erva-doce, cordão de São } \\
\text { Francisco; massagens; uso de objetos e adereços de cunho protetivo espiritual; } \\
\text { crenças e comportamentos relacionados ao movimento do corpo: não sentar } \\
\text { em pedra ou em pilão, não saltar corda de animal, não pegar peso; práticas de } \\
\text { resguardo no pós-parto e pós-aborto; práticas de repouso e higiene no puerpério. }\end{array}$ \\
\hline $\begin{array}{l}\text { Conhecimento } \\
\text { do corpo, } \\
\text { autoestima e } \\
\text { protagonismo }\end{array}$ & $\begin{array}{l}\text { Valorização estética e autoestima das mulheres. Comportamentos de valorização } \\
\text { de seus traços de mulher negra; valorizam tranças, turbantes, maquiagem, roupas } \\
\text { de estilo afro; participam de ações culturais com danças e músicas relacionadas } \\
\text { com a cultura negra; as mulheres demonstram conhecimento do corpo e da } \\
\text { contagem do tempo gestacional e sinais e sintomas do parto que as ajudam no } \\
\text { processo de autocuidado nas iminências de busca por cuidados profissionais; } \\
\text { avaliação positiva de saúde centrada nos cuidados populares. }\end{array}$ \\
\hline $\begin{array}{l}\text { Aspectos } \\
\text { relacionais, } \\
\text { comunitários e } \\
\text { políticos }\end{array}$ & $\begin{array}{l}\text { Rede de solidariedade (ajuda da família, vizinhos, lideranças comunitárias e } \\
\text { espirituais); envolvimento familiar nas orientações para a mulher gestante; } \\
\text { formação, organização e mobilização política da comunidade mediante } \\
\text { associação comunitária quilombola, com reuniões e discussões de problemas } \\
\text { comunitários de forma sistemática e periódica; aspectos éticos de compromissos } \\
\text { e responsabilidades do cuidado da família e da comunidade. }\end{array}$ \\
\hline
\end{tabular}

Fonte: Elaboração própria.

Os aspectos culturais observados relativos ao conhecimento do corpo, autocuidado nas proximidades do parto, autonomia e protagonismo de muitas mulheres no momento do parto são de grande relevância e requerem preservação, ações para aumentar os estímulos e capacitações para aprofundamentos na comunidade estudada, tendo em vista a influência das questões de gênero na saúde. Essa perspectiva favorece a desconstrução do viés de gênero, entendido como um determinante biológico que impede as mulheres de terem controle sobre seus próprios corpos e de fazerem escolhas conscientes relacionadas à sua sexualidade e saúde. Portanto, conhecimento e protagonismo das mulheres possibilitam o enfrentamento de situação de subalternidade nas relações existentes entre profissionais e usuárias ${ }^{(9)}$.

Já a utilização de plantas e animais para chás, banhos e rezas e ainda a perspectiva de restrição e recomendações de determinados alimentos em períodos específicos da experiência em saúde reprodutiva refletem a presença da medicina tradicional, com seus princípios de cura. Além disso, mostra também a cultura das mulheres quilombolas, a cultura de um povo e sua memória, indicando a ligação dessa forma de cuidado com o modo de vida relacionado 
ao meio ambiente, com um mundo natural. Essa ligação é evidenciada quando animais e plantas são transformados em medicamentos produzidos de diversas formas, a exemplo de garrafadas, lambedores, chás para banho ou ingestão e rezas $^{(10)}$. O pensar na preservação dessa forma de cuidado está imbricado com a valorização da experiência e do respeito à forma de viver de diversas comunidades, que aprenderam com seus ancestrais, com base na transmissão oral, práticas de cuidado que expressam resultados positivos, crenças, comportamentos e atitudes.

A importância da religiosidade no conforto e bem-estar das mulheres foi um aspecto observado. O constructo de cuidado cultural engendrado com base em rezas, benzimentos e crenças espirituais, observados no modo de vida de muitas mulheres quilombolas, nos aspectos de prevenção, promoção da saúde e cuidados em saúde reprodutiva, propicia pensar a preservação, tendo em vista o entendimento da religião como um aporte de cura e de cuidados. Nessa perspectiva, os atendimentos, as benzeduras e a proteção operam sentidos ampliados do entendimento de saúde e produzem uma lógica sociocosmopolítica das curas e das relações. Os espaços de cura têm lugar para a religiosidade como intermediadores para o cuidado ${ }^{(11)}$.

Os aspectos observados de rede de solidariedade, envolvimento familiar, ética e responsabilidade do cuidado remetem ao pensamento do conceito de quilombo enquanto comunidade em solidariedade, em convivência e comunhão existencial $^{(12)}$. Essa perspectiva que direciona a proposta de preservação do cuidado, espelhada na atenção recebida nos cuidados familiares e de vizinhança pelas mulheres quilombolas em suas necessidades nos diversos momentos da vida sexual e reprodutiva, precisa ser preservada no cotidiano do cuidado de enfermagem e dos serviços. Apesar de se configurar como um princípio do Sistema Único de Saúde, observam-se, na prática, aspectos e relações de distanciamento entres usuários e profissionais.

Logo, entende-se a solidariedade como reciprocidade ou interdependência, e essa reciprocidade mantém as relações sociais fortalecidas, além de possibilitar a assistência entre os membros de um grupo, assegurando coesão interna ${ }^{(13)}$. Além disso, entende-se que a solidariedade possui valor terapêutico, ao manter o compromisso e a responsabilidade de uma rede funcionando de forma articulada, com corresponsabilização entre os diversos serviços e níveis de saúde, assim como entre profissionais, usuários e sociedade. Assim, a solidariedade torna possível avivar uma determinada rede de atenção.

A organização e a mobilização políticas observadas no seio da comunidade, da qual participam muitas mulheres do estudo, é um aspecto de grande valor para a preservação do cuidado cultural. Nesse sentido, chama-se a atenção de profissionais de saúde para refletirem nessa experiência que possibilita o enfrentamento de vulnerabilidades em saúde e para a aprendizagem sobre participação política, tão necessários no contexto do cuidar em saúde.

Afirma-se, em concordância com o achado de outro estudo ${ }^{(14)}$, que a gestação e a parturição estão entrelaçadas por construções históricas, culturais e com abordagem de gênero. Por isso, é necessário atentar para as subjetividades e para a identidade particularizada de cada mulher, levantando seus valores para propiciar coerência no cuidado.

\section{Proposições de acomodação e/ou negociação do cuidado cultural}

No Quadro 2, são descritos os cuidados ou aspectos valorizados pelas mulheres, potencialmente benéficos, mas com interface com vulnerabilidades para adoecimento, com reflexões para a ação profissional de negociação do cuidado. 
Quadro 2 - Aspectos culturais do cuidado popular em saúde reprodutiva de mulheres quilombolas com reflexões para a ação de negociação do cuidado

\begin{tabular}{|l|l|}
\hline Subcategorias & \multicolumn{1}{c|}{ Aspectos culturais observados } \\
\hline $\begin{array}{l}\text { Cuidados } \\
\text { alimentares }\end{array}$ & $\begin{array}{l}\text { Alimentação com mariscos e azeite com sinalização de relação com hipertensão } \\
\text { nas gestantes; descrição de alimentação inadequada na gestação, incorrendo em } \\
\text { diversas gestantes com quadros de anemia. }\end{array}$ \\
\hline $\begin{array}{l}\text { Relação com } \\
\text { cuidados } \\
\text { profissionais }\end{array}$ & $\begin{array}{l}\text { As mulheres demonstravam desconfiança quanto à qualidade das medicações } \\
\text { fornecidas para contracepção. Queixavam-se de déficit de cuidados } \\
\text { ginecológicos. Muitas desconheciam métodos contraceptivos. Muitas mulheres } \\
\text { jovens são grandes multíparas, com mais de 5 filhos; baixa procura pelo serviço } \\
\text { de saúde no pós-parto; restrição de serviços ginecológicos e de planejamento } \\
\text { reprodutivo; descrição das mulheres sobre a demora nos resultados de exames } \\
\text { preventivos, citopatológicos. }\end{array}$ \\
\hline $\begin{array}{l}\text { Terapias } \\
\text { complementares }\end{array}$ & $\begin{array}{l}\text { Dosagem, causas e efeitos das ervas utilizadas durante a gestação na forma de } \\
\text { chás para uso oral; partos acidentais, com risco de intercorrências obstétricas } \\
\text { e neonatais. As mulheres queixavam-se de falta de parteiras e de serviços } \\
\text { resolutivos próximos à comunidade; algumas ervas medicinais precisavam ser } \\
\text { compradas, denotando perda de recursos culturais. }\end{array}$ \\
\hline $\begin{array}{l}\text { Barreiras e } \\
\text { vulnerabilidades } \\
\text { socioambientais }\end{array}$ & $\begin{array}{l}\text { Trabalho realizado por gestantes na maré ou na agricultura, em meio às } \\
\text { intercorrências na gestação; jornada tripla de trabalho; dificuldade de acesso a } \\
\text { transporte para os serviços de saúde. }\end{array}$ \\
\hline
\end{tabular}

Fonte: Elaboração própria.

A alimentação com mariscos e azeite de dendê é uma rotina na vida das mulheres estudadas. Tendo em vista esses alimentos fazerem parte da cultura ambiental da comunidade, que se situa às margens do Rio Paraguaçu, é importante negociar esse aspecto com as mulheres, tendo em vista que as preferências alimentares e a condição de acesso aos alimentos devem ser consideradas no processo de orientação alimentar ${ }^{(15)}$. No controle da hipertensão relacionada com alimentos, a literatura científica refere frutos do mar (camarão, ostra, marisco, lagosta) como fonte de colesterol e o azeite de dendê como alimento fonte de ácidos graxos saturados. Os alimentos saturados em gorduras e ricos em colesterol têm influência direta e diversa nos níveis de lipídios na circulação, sobretudo a colesterolemia. Esses alimentos devem ser evitados, nos casos de dislipidemias, porquanto as alterações das gorduras sanguíneas influenciam na elevação da pressão $\operatorname{arterial}^{(16)}$.

A hipertensão arterial sistêmica tem definição bem consolidada. Trata-se de uma patologia de etiologia múltipla, caracterizada por elevação dos níveis pressóricos com consequentes impactos em funções e estruturas de órgãos importantes como rins, coração, vasos sanguíneos e encéfalo, além de impactos no metabolismo orgânico ${ }^{(16)}$.

A hipertensão arterial e a anemia são apontadas por profissional de saúde da comunidade como problemas que têm acometido as mulheres e estão relacionadas com o fator alimentar. Entretanto, outros aspectos sociais e econômicos podem também estar relacionados ao aparecimento de hipertensão nas mulheres. Por isso, essa demanda necessita de outros estudos e aprofundamentos. Em outras comunidades quilombolas, a hipertensão tem chamando a atenção enquanto problema de saúde e relacionado a fatores como condições socioeconômicas ${ }^{(1)}$. De qualquer modo, discussões e negociações com vistas a melhorias no estilo de vida, independentemente da causa, controle na ingestão de azeite de dendê e mariscos devem ser tentadas para prevenir novos casos e cuidar das mulheres que já apresentam a doença. 
No que tange à anemia, mesmo que as mulheres implementem o cuidado popular do uso de vegetais conhecidamente ricos em ferro, propõe-se a negociação do cuidado, a fim de a enfermeira e outros profissionais propiciarem a facilitação do uso de uma dieta diversificada equilibrada, bem como a reorientação de suplementação de nutrientes necessários. Além disso, seria indicado investigar, de forma mais profunda, a ocorrência desse problema entre essas mulheres.

Aspectos observados e ditos pelas mulheres relacionados aos serviços de atenção à saúde da mulher, a exemplo das desconfianças em relação à qualidade de métodos contraceptivos fornecidos, deficiência na oferta de cuidados ginecológicos e de planejamento reprodutivo, queixas quanto à demora no retorno de resultados de exames colpocitológicos (preventivo), configuram facetas da vulnerabilidade política a ser enfrentada pelas quilombolas e pelos serviços de saúde, sendo a enfermeira uma profissional-chave nessa interface. A baixa procura das mulheres pelo serviço no pós-parto e o desconhecimento apontado por elas sobre métodos de prevenção da gestação, somado à situação de multiparidade, sobretudo de mulheres jovens, sinalizam necessidades de negociações com as políticas de saúde local, para o desenvolvimento de melhores suportes assistenciais em saúde reprodutiva e sexual para as quilombolas.

A referida vulnerabilidade política está relacionada às ações públicas de enfrentamento de problemas e agravos, metas e ações propostas nos diversos programas e organizações governamentais ou não, bem como à distribuição dos recursos para prevenção e controle de problemas e agravos. Controle social, definição de políticas específicas, sustentabilidade e avaliação da política, articulação e atividades intersetoriais, integralidade da atenção, qualidade dos serviços, preparo tecnocientífico dos profissionais e das equipes, compromisso e responsabilidade dos profissionais, respeito, proteção e promoção dos direitos humanos são exemplos de fatores que permitem avaliar esse tipo de vulnerabilidade ${ }^{(17)}$.
Entre as mulheres estudadas, é bastante comum o uso do chá de erva-doce durante a gravidez. A erva-doce é utilizada culturalmente, há muito tempo. Entretanto, apontamentos teóricos referentes ao uso dessa erva na gestação relaciona-a com a possibilidade de intercorrências. Por isso, é contraindicada no período gravídico ${ }^{(18)}$. Por essa razão, é importante negociar com as mulheres uma capacitação, para direcionar uma utilização de forma segura, aprofundando estudos sobre dosagens que possam ser terapêuticas, sem prejuízo para a gestante e o concepto. Essa medida dará mais segurança à enfermeira para realizar orientações apoiadas na lógica do uso racional $^{(19)}$.

O uso de ervas, como o cordão de São Francisco e o cajueiro branco, na forma de banhos e chás no final da gestação, para acelerar o parto, é um cuidado cultural bem conhecido entre as mulheres estudadas. Essa tecnologia, que muitas parteiras usufruíam no seu trabalho cotidiano, proporcionava partos rápidos. Contudo, a continuidade desse cuidado, na ocorrência de ausência de parteiras e na perspectiva de acesso limitado ao transporte, pode explicar a ocorrência do parto acidental. Algumas mulheres, inclusive, relataram que utilizavam esse cuidado para que a permanência no serviço hospitalar fosse a mínima possível, diante da assistência desumanizada recebida nesse ambiente.

À vista desse processo e das possibilidades de intercorrências do parto acidental, sem acesso a recursos humanos locais para prestar uma assistência adequada, propõe-se a negociação desse cuidado, de forma a permitir maior segurança para a mulher e seu filho. Entretanto, o cuidado popular considerado efetivo não deve ser esquecido e menosprezado pelas mulheres. Ele pode, sim, ser discutido para sua ampliação e uso racional, incentivando a implementação em situações em que haja pessoas da comunidade com destreza, orientação e protagonismo para cuidar/ ajudar a mulher nesse momento ou dentro do ambiente profissional de cuidados. Nesse aspecto, a proposta da negociação é também para o serviço de saúde, que pode manter seus cuidados técnicos e permitir o protagonismo da 
10

Proposições de cuidado cultural à enfermagem frente a aspectos da saúde reprodutiva de mulheres quilombolas

mulher, valorizando sua experiência de uso de terapia popular para facilitar o trabalho de parto, tornando a experiência mais agradável, dinâmica, participativa e humana ${ }^{(20)}$.

Algumas ervas, como poejo, losna, palma e cebola branca, necessitam ser compradas no comércio local. Algumas mulheres relataram que também podiam ser encontradas na flora local. Nessa perspectiva, vê-se a necessidade de articular com elas formas de revitalização e recuperação de recursos vegetais, discutindo sobre replantio, para favorecer o fortalecimento e a preservação do arcabouço cultural. Essa dinâmica pode, inclusive, ser ampliada para fomentar ação empreendedora e fonte econômica para a comunidade, que possui solo propício para cultivos agrícolas diversos.

Não se pode ignorar, entretanto, que os manguezais brasileiros estão ameaçados, perdendo sua potência, pois tem sido observada invasão das áreas por indústrias, ações poluentes e alterações na dinâmica de obtenção de recursos hídricos. Esses fatores acabam resultando em rupturas e perdas da cobertura vegetal, vulnerabilidades e riscos à sobrevivência de muitas comunidades tradicionais ${ }^{(21)}$.

Outro aspecto identificado no cotidiano das mulheres que participaram deste estudo, que perpassa a saúde reprodutiva, é o uso do tempo e o trabalho, que fazem interseção com a classe social. Movidas pelas necessidades de cuidado da família, elas empreendiam uma grande força de trabalho durante a gestação, dentro e fora do ambiente doméstico, relatando, em muitas situações, o cansaço físico. Além do trabalho como marisqueiras, pescadoras e agricultoras, usavam grande parte do tempo no trabalho de reprodução social, cuidando dos afazeres domésticos, filhos, companheiros e outras demandas.
Observam-se, nesse contexto, duplas, triplas, amplas jornadas de trabalho, caracterizando desigualdades de gênero também para as mulheres quilombolas. Diversos estudos, que têm abordado o uso do tempo nas dinâmicas entre trabalho e vida cotidiana das mulheres, afirmam que esse diagnóstico injusto deve direcionar ações de movimentos sociais e gestores de políticas públicas para construções de agendas que pautem a igualdade e o conhecimento dessa vulnerabilidade social de forma ampla nos diversos setores da sociedade ${ }^{(22)}$.

Tratando-se de mulheres grávidas, essas situações podem sinalizar prejuízos à saúde, incorrendo nos riscos durante a gravidez, causando precocidade do parto e problemas na saúde e no desenvolvimento da criança. A literatura científica nacional refere a importância de rotina de trabalho sem sobrecargas e com intervalo de descanso dignos. Os riscos à saúde podem ser minimizados, já que, nesse caso, 8 horas de trabalho diárias deve ser o escopo máximo. Além disso, o direito à licença maternidade, possibilita à mulher melhor condição de cuidar do recém-nascido, pode impedir a ocorrência de adoecimento crônico e a mortalidade materna ${ }^{(5,22)}$.

\section{Proposições de decisões e ações de reestruturação ou repadronização do cuidado de enfermagem}

Os aspectos que significaram dificuldades e vulnerabilidades, sofrimentos ou problemas e não expressaram benefícios para a vida e a saúde foram designados como modo de ação de reestruturação de cuidados e estão descritos no Quadro 3. 
Quadro 3-Aspectos culturais do cuidado popular em saúde reprodutiva de mulheres quilombolas com reflexões para ação de repadronização ou reestruturação do cuidado

\begin{tabular}{|l|l|}
\hline Subcategorias & \multicolumn{1}{c|}{ Aspectos culturais observados } \\
\hline $\begin{array}{l}\text { Relação com cuidados } \\
\text { profissionais }\end{array}$ & $\begin{array}{l}\text { Gestantes com recebimento de alta do serviço de pré-natal; início tardio } \\
\text { do pré-natal, devido às barreiras socioambientais e de acesso aos serviços; } \\
\text { experiências e histórias de violência obstétrica institucionalizada no parto e } \\
\text { aborto; ocorrências de aborto inseguro; experiências e histórias de racismo } \\
\text { institucional relatadas pelas mulheres quilombolas. }\end{array}$ \\
\hline $\begin{array}{l}\text { Barreiras } \\
\text { socioambientais }\end{array}$ & $\begin{array}{l}\text { Ocorrências de gravidez indesejada; histórias de gravidez na adolescência } \\
\text { e abandono escolar; baixo nível educacional; muitas mulheres jovens não } \\
\text { completam o ensino fundamental e/ou médio. Algumas revelam desejo } \\
\text { de continuação dos estudos; desigualdade de gênero no trabalho, jornada } \\
\text { dupla ou tripla e contínua de atividades; ocultamento de algumas mulheres } \\
\text { quanto à busca por cuidados em religiões de matriz africana; relato das } \\
\text { mulheres marisqueiras de prurido corporal constante após o contato com a } \\
\text { água e a lama do mangue. }\end{array}$ \\
\hline
\end{tabular}

Fonte: Elaboração própria.

O serviço de saúde necessita avaliar as situações de ocorrência de liberação/alta das gestantes do serviço de pré-natal, tendo em vista que políticas que orientam ação profissional na atenção primária em saúde referem a perspectiva de retaguarda e maior constância de consultas no final da gestação, para melhor avaliação e seguimento do risco perinatal e das intercorrências clínico-obstétricas mais comuns nesse período ${ }^{(3)}$. Portanto, esse comportamento profissional necessita ser repadronizado, por se mostrar inadequado para prover mais conforto e segurança para as mulheres, ao contrário da alta, que desvincula a mulher do serviço. Desse modo, torna-se necessário organizar ações de ampliação dos vínculos com o serviço e acompanhá-la com acolhimento e classificação de risco contínuos, para melhorar a qualidade do cuidado ${ }^{(3,23)}$.

O início tardio do cuidado pré-natal, já reconhecido como um fator de risco para complicações maternas e fetais, potencializa as inadequações. Esse fato demanda ações multiprofissionais de articulação de programas e serviços de saúde, na perspectiva de favorecer impactos positivos na saúde materno-infantil em populações com vulnerabilidade de cuidados ${ }^{(3,23-24)}$.

As condições de discriminações raciais e de gênero, associadas às experiências de violência obstétrica no trabalho de parto e nos processos de aborto, historicizadas pelas próprias mulheres, dão créditos para o planejamento de ações e decisões de reestruturação da abordagem profissional na atenção à saúde das mulheres quilombolas. Essas observações e informações das mulheres confirmam apontamentos acerca da realidade do cuidado institucional dispensado às mulheres, denunciados por diversos autores ${ }^{(1,5,20)}$. Apesar das inovações nas políticas de atenção à saúde das mulheres brasileiras, que reconhecem o racismo e o sexismo como determinantes sociais em saúde, nos últimos dez anos, a realidade aponta necessidades urgentes e contínuas de enfrentamentos ${ }^{(4)}$.

Para tanto, a formação política e de empoderamento das mulheres é premente para efetivo exercício do controle social. Assim, na perspectiva da reestruturação do cuidado, propõe-se que enfermeiras das unidades de saúde da família e enfermeiras relacionadas com as comunidades pelo vínculo das pesquisas possam articular capacitações comunitárias sobre formação política, racismo, desigualdades de gênero e empoderamento das mulheres apoiadas em metodologias participativas.

Esses temas necessitam ser dimensionados também nas reuniões da equipe de saúde, para que, além disso, possam articular a formação de uma rede de cuidados baseada nas dificuldades 
12

Proposições de cuidado cultural à enfermagem frente a aspectos da saúde reprodutiva de mulheres quilombolas

apresentadas pelas mulheres. Essa rede deve contemplar a assistência localizada, articulação para criação de uma casa de parto nas comunidades quilombolas e ainda a formação de um observatório da violência e das desigualdades de gênero e raciais, com abordagens intersetoriais ${ }^{(5)}$.

Essas propostas podem ser desenhadas com maior participação das mulheres e o apoio das associações comunitárias já existentes nas comunidades. No processo de observação da comunidade, notou-se que há pouca articulação de enfermeiras e de outros profissionais de saúde com esses mecanismos sociais. Esse aspecto pode ser vitalizado, estruturado, pois o enfrentamento dessas desigualdades sociais a favor da comunidade será possível pela perspectiva do envolvimento político e por meio dos movimentos sociais ${ }^{(4,12-13,17)}$.

A formação política e o empoderamento das mulheres poderão contribuir para uma mudança no quadro social apresentado no nível de escolarização. É possível elevar os escores educacionais dessas mulheres, inclusive com ações efetivas de prevenção da gravidez na adolescência, que é traduzida como um fator associado à evasão escolar $^{(25)}$. A associação comunitária permite à enfermeira articular com a Secretaria de Educação Municipal, para promover cursos locais de educação de jovens e adultos para complementação do ensino médio e fundamental, além de políticas que entusiasmem os jovens para a formação superior.

Observou-se o empreendimento de dinâmicas de trabalho que potencializam a região de mangue pela comunidade, a exemplo do cultivo de ostra, do dendê e da mandioca. Entretanto, verificou-se a existência de grandes áreas sem cultivo de outros produtos agrícolas de grande potencial na região, que poderiam gerar fontes de renda para as mulheres na comunidade. Tendo em vista que as dificuldades socioeconômicas influenciam sobremaneira na saúde reprodutiva e na saúde geral, é de extrema importância o incentivo e a articulação com profissionais técnicos especializados que fomentem a ampliação de cultivos agrícolas e o empreendedorismo para essa comunidade, de forma que os jovens identifiquem condições de sobrevivência para continuar morando na localidade ${ }^{(21)}$.

Outrossim, é preciso reestruturar o cuidado com a saúde da mulher trabalhadora. A ocorrência de pruridos e outras reações orgânicas em decorrência do contato com a água do rio sinalizam perda das condições naturais de vitalidade e ocorrência de contaminação por produtos químicos e físicos, denunciando degradação do meio ambiente ${ }^{(21)}$. Os profissionais de saúde, responsáveis pela comunidade, necessitam estar articulados com a situação de saúde apresentada. Para isso, é importante estruturar cuidados com ações de pesquisa para desvendar as causas do problema. Nesse sentido, propõe-se a reestruturação do cuidado, para organizar, capacitar e fornecer equipamentos de proteção individuais e coletivos que possam prevenir agravos e promover a saúde das mulheres quilombolas.

Esta proposta inicial de cuidado cultural tem suas limitações, por não estar finalizada com proposições e discussões mais ampliadas com as mulheres e suas famílias. Contudo, elas apontaram as questões direcionadas para ações de preservação, negociação ou reestruturação do cuidado. Reafirma-se que são poucos os artigos com aplicabilidade da Teoria do Cuidado Cultural e que puderam ser utilizados na discussão deste trabalho. Entretanto, a Enfermagem vem utilizando essa teoria nas diversas áreas de sua atuação e em diferentes temáticas, fato de interesse para valorização e avanços da enfermagem transcultural.

\section{Conclusão}

Cuidados alimentares, terapias complementares, conhecimento do corpo, aspectos relacionais, comunitários e políticos e relações com os serviços profissionais são experimentados pelas mulheres quilombolas. Muitos aspectos amparam benefícios à saúde e outros potencializam vulnerabilidades para o adoecimento, como reconhecem as mulheres. Com isso, propõe-se o desenvolvimento de ações de enfermagem com vistas à preservação, negociação e repadronização do cuidado para favorecer maior 
empoderamento das mulheres no cuidado em saúde reprodutiva.

Este artigo responde ao nível quatro da Teoria Transcultural do Cuidado. Reflete-se que muitas ações podem ser implementadas na interface do cuidado cultural com as mulheres quilombolas estudadas, com vistas às transformações dos serviços e de práticas cotidianas realizadas por elas. Uma grande frente de atividades é vislumbrada, envolvendo cuidados assistenciais diretamente ligados à saúde, mas que abrange ações relacionadas com o campo de construções e de enfrentamentos políticos que impactam na saúde, além de enfrentamento de vulnerabilidades sociais, abarcando desigualdades de gênero, de classe, raciais e geracionais.

Reflete-se ainda sobre a complexidade que está presente quando se propõem decisões e ações de cuidado cultural excluindo o etnocentrismo. Além disso, sobre a complexidade da proposição de ações, enquanto pesquisadora que não está implicada no cuidado assistencial com a comunidade, de forma direta. Entretanto, a realização deste estudo etnográfico configura-se como um suporte para abertura de uma linha de pesquisa na academia, possibilitando tanto a introdução da temática na formação curricular de novas enfermeiras quanto o amadurecimento de novas investigações e extensões universitárias que possam colaborar com o cuidado cultural à saúde reprodutiva das mulheres quilombolas. Favorece também a articulação com o serviço de saúde local, ao demandar suas necessidades.

Conclui-se que as proposições de cuidado cultural para a enfermagem frente a aspectos da saúde reprodutiva de mulheres quilombolas rurais podem favorecer maior empoderamento dessas mulheres e o enfrentamento de vulnerabilidades sociais.

\section{Colaborações:}

1 - concepção, projeto, análise e interpretação dos dados: Amália Nascimento do Sacramento Santos e Enilda Rosendo do Nascimento;

2 - redação do artigo e revisão crítica relevante do conteúdo intelectual: Amália
Nascimento do Sacramento Santos e Enilda Rosendo do Nascimento;

3 - aprovação final da versão a ser publicada: Amália Nascimento do Sacramento Santos e Enilda Rosendo do Nascimento.

\section{Referências}

1. Bezerra VM, Medeiros DS, Gomes KO, Souzas R, Giatti L, Stefens AP, et al. Inquérito de saúde em comunidades Quilombolas de Vitória da Conquista/BA: aspectos metodológicos e análise descritiva. Ciênc saúde coletiva. 2014;19(6):183547. DOI: 10.1590/1413-81232014196.01992013

2. Veliz-Rojas L, Bianchetti-Saavedra AF, SilvaFernández M. Competências interculturais na atenção primaria à saúde: um desafio para a educação superior frente a contextos de diversidade cultural. Cad Saúde Pública. 2019;35(1):e00120818. DOI: 10.1590/0102-311X00120818

3. Domingues RMSM, Viellas EF, Dias MAB, Torres JA, Theme-Filha MM, Gama SGN, et al. Adequação da assistência pré-natal segundo as características maternas no Brasil. Rev Panam Salud Publica [Internet]. 2015 [cited 2016 Jan 22];37(3):140-7. Disponível em: http://portaldeboaspraticas. iff.fiocruz.br/wp-content/uploads/2018/07/ v37n3a03.pdf

4. Durand MK, Heideman ITSB. Determinantes sociais de uma comunidade quilombola e a interface com a Promoção da Saúde. Rev esc enferm USP. 2019;53:e03451. DOI: 10.1590/ s1980-220x2018007703451

5. Diniz SG, Salgado HO, Andrezzo HFA, Carvalho PGC, Carvalho PCA, Aguiar CA, et al. Violência obstétrica como questão para a saúde pública no Brasil: origens, definições, tipologia, impactos sobre a saúde materna, e propostas para sua prevenção. J Hum Growth Dev. 2015;25(3):37784. DOI: $10.7322 /$ jhgd. 106080

6. Castillo LO, López-Díaz L. Competencia cultural de enfermeras en salud pública con población indígena. Av enferm. 2019;37(1):9-18. DOI: 10.15446/av.enferm.v37n1.68513

7. Leininger MM, Mcfarland MR. Culture care diversity and universality: a worldwide nursing theory. 2a ed. New York: Jones and Bartlett Publishers; 2006.

8. Leopardi MT. Teorias em enfermagem: instrumentos para a prática. Florianópolis: Papa-Livros; 1999. 
9. Silva ALS, Nascimento ER, Coelho EAC. Práticas de enfermeiras para promoção da dignificação, participação e autonomia de mulheres no parto normal. Esc Anna Nery. 2015;19(3):424-31. DOI: 10.5935/1414-8145.20150056

10. Kagan CMC. E quando a cura vem da mata? Em forma de planta e em forma de bicho! Um olhar antropológico sobre a medicina tradicional dos Pitaguary de Monguba, suas técnicas e seus rituais. In: Andrade JT, Melo ML, Holanda VMS. Saúde e cultura: diversidades terapêuticas e religiosas. Fortaleza: EDUECE; 2015. p. 233-53.

11. Ramos JDD. A religião é como uma medicina. Aprontamento, cosmopolítica e cura entre umbandistas em Mostardas, Rio Grande do Sul. In: Andrade JT, Melo ML, Holanda VMS. Saúde e cultura: diversidades terapêuticas e religiosas. Fortaleza: EDUECE; 2015. p. 22-55.

12. Nascimento A. Uma mensagem do quilombismo. In: Nascimento A, organizador. O negro revoltado. 2a ed. Rio de Janeiro: Nova Fronteira; 1982. p. 24-35.

13. Turcato JA. A solidariedade como um postulado da razão comunicativa e da ética do discurso. Thaumazein [Internet]. 2010 [cited 2015 Mar 17]; 3(6):50-68. Available from: https://periodicos.ufn. edu.br/index.php/thaumazein/article/view/163/ pdf

14. Camargo FCM, Lima RFS, Santos AM, Silva LR, Santos IMM. A aplicabilidade da teoria do cuidado cultural por enfermeiras nos periódicos de saúde do Brasil (1992-2011). Rev pesqui cuid fundam. 2014;6(4):1743-55.

15. Brasil. Ministério da Saúde. Secretaria de Atenção à Saúde. Departamento de Atenção Básica. Guia alimentar para a população brasileira. 2a ed. Brasília, DF; 2014.

16. Malachias MVB, Souza WKSB, Plavnik FL, Rodrigues CIS, Brandão AA, Neves MFT, et al. $7^{\text {a }}$ Diretriz Brasileira de Hipertensão Arterial. Arq Bras Cardiol [Internet]. 2016 [cited 2016 Apr 20];107(3supl3):1-83. Available from: http:// publicacoes.cardiol.br/2014/diretrizes/2016/05_ HIPERTENSAO_ARTERIAL.pdf
17. Oviedo RAM, Czeresnia D. O conceito de vulnerabilidade e seu caráter biossocial. Interface (Botucatu). 2015;19(53):237-50

18. Gonçalves KG, Pasa MC. Saber local e as plantas medicinais na comunidade Sucuri, Cuiabá, MT, Brasil. Biodiversidade. 2015;14(2):50-73.

19. Rocha BS, Werlang MC. Psicofármacos na Estratégia Saúde da Família: perfil de utilização, acesso e estratégias para promoção do uso racional. Ciênc saúde coletiva. 2013;18(11):3291-300.

20. Watson H, Harrop D, Walton E, Young A, Soltani H. A systematic review of ethnic minority women's experiences of perinatal mental health conditions and services in Europe. PLoS One. 2019;14(1):e0210587. DOI: 10.1371/journal. pone. 0210587

21. Instituto Chico Mendes de Conservação da Biodiversidade. Atlas dos Manguezais do Brasil. Brasília; 2018.

22. Bandeira LM, Petrurlan RB. As pesquisas sobre uso do tempo e a promoção da igualdade de gênero no Brasil. In: Fontoura N, Araújo C, Barajas MPL, organizadoras. Uso do tempo e gênero. Rio de Janeiro: UERJ; 2016. p. 43-60.

23. Cassiano ACM, Carlucci EMS, Gomes CF. Saúde materno infantil no Brasil: evolução e programas desenvolvidos pelo Ministério da Saúde. Rev Serv Público. 2014;65(2):227-44.

24. Hernández-Vásquez A, Vargas-Fernández R, Bendezu-Quispe G. Factors associated with the quality of prenatal care in Peru. Rev Peru Med Exp Salud Publica. 2019;36(2):178-87. DOI: 10.17843/ rpmesp.2019.362.4482

25. Sousa CRO, Gomes KRO, Silva KCO, Mascarenhas MDM, Rodrigues MTP, Andrade JX, et al. Fatores preditores da evasão escolar entre adolescentes com experiência de gravidez. Cad saúde colet. 2018;26(2):160-9.

Recebido: 30 de agosto de 2019 Aprovado: 4 de novembro de 2019 Publicado: 4 de fevereiro de 2020 


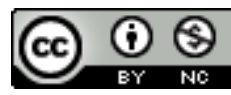

A Revista Baiana de Enfermagem utiliza a Licença Creative Commons - Atribuição-NãoComercial 4.0 Internacional. https://creativecommons.org/licenses/by-nc/4.0/ Este artigo é de acesso aberto distribuído sob os termos da Licença Creative Commons (CC BY-NC). Esta licença permite que outros remixem, adaptem e criem a partir do seu trabalho para fins não comerciais. Embora os novos trabalhos tenham de lhe atribuir o devido crédito e não possam ser usados para fins comerciais, os usuários não têm de licenciar esses trabalhos derivados sob os mesmos termos. 